\title{
INADIMPLÊNCIA NAS COOPERATIVAS DE CRÉDITO DE LIVRE ADMISSÃO
}

\author{
DEFAULT IN CREDIT COOPERATIVES WITHOUT COMMON BOND TYPE
}

\author{
${ }^{a}$ Romeu Eugênio Lima; ${ }^{b}$ Hudson Fernandes Amaral \\ ${ }^{a}$ Analista do Banco Central do Brasil, BACEN; Doutorado em andamento em Administração pela Universidade Federal de Minas Gerais, UFMG; Mestrado em \\ Administração pela Universidade Federal de Minas Gerais, UFMG, Belo Horizonte, MG-Brasil; E-mail: romeulima71@yahoo.com.br \\ ${ }^{b}$ Professor da Universidade Federal de Minas Gerais - UFMG; Doutorado em Sciences de Gestion pela Universite Pierre Mendes \\ France Grenoble II Franca; Belo Horizonte, MG - Brasil; E-mail: hfamaral@Cepead.face.ufmg.br
}

\section{Resumo}

Nos últimos anos, ocorreram importantes alterações normativas no Brasil relacionadas ao cooperativismo de crédito. Merece destaque a edição da Resolução CMN 3.106/03, que permitiu a criação de cooperativas de crédito de livre admissão, e a transformação das cooperativas de crédito existentes nesta nova modalidade. As cooperativas de crédito estão entre as instituições financeiras menos estudadas no Brasil e, de modo geral, é grande o desconhecimento sobre o tema. Neste contexto, este artigo procurou estudar a importância das cooperativas de crédito e o desempenho do setor com foco na inadimplência de suas carteiras de crédito. Finalmente, o Modelo de Equações de Estimação Generalizadas (GEE) é utilizado, a fim de verificar se houve alguma mudança nesse indicador que pudesse ser atribuída à transformação das cooperativas de crédito para a modalidade de livre admissão, ao sistema cooperativista de crédito a que pertença ou ao seu tamanho.

Palavras-chave:Cooperativismo de crédito, inadimplência, Modelo de Equações de Estimação Generalizadas (GEE)

\section{Abstract}

In the last years, it has took place important rule changes in Brazil related with credit cooperativism. It can be detached the edition of Resolution CMN 3.106/03 that allowed the creation of new credit cooperatives without common bond type and the transformation of existing ones into this new type. Furthermore, the credit cooperatives are among financial institutions less studied and it is great the misunderstanding in Brazil about them. In this context, this paper studied credit cooperativism importance and performance with focus on portfolios default. Then, Generalized Estimation Equations Model (GEE) is used to determine if it happened performance changes in default of credit cooperative portfolios that transformed themselves into cooperatives without common bond type.

Keywords: Credit union, credit unions, free admission, delinquency rate, Model Generalized Estimating Equations (GEE). 


\section{INTRODUÇÃO}

Nos últimos anos, ocorreram no Brasil importantes alterações normativas relacionadas ao cooperativismo de crédito. Merece destaque a edição da Resolução CMN 3.106, de 25 de junho de 2003, que permitiu a criação de cooperativas de crédito de livre admissão, e a transformação das cooperativas de crédito existentes nesta nova modalidade. Dessa forma, poderão se associar à cooperativa de crédito quaisquer pessoas físicas ou jurídicas que residam na área de atuação estabelecida em seu Estatuto Social.

Outro aspecto importante é que, segundo Cuevas e Fischer (2006), as cooperativas de crédito estão entre as instituições financeiras menos estudadas. Além disso, Pinheiro (2007) constata que é grande o desconhecimento sobre o cooperativismo de crédito no Brasil tanto pelo público em geral como por muitos estudiosos em finanças.

Neste contexto, este trabalho procurou estudar a inadimplência das carteiras de crédito das cooperativas de crédito que se transformaram para a modalidade de livre admissão. Inicialmente, procurou-se contextualizar o problema de pesquisa a partir de um referencial teórico que focasse o sistema financeiro e o cooperativismo de crédito. O desempenho de cooperativas de crédito é, então, discutido.

Em seguida, o Modelo de Equações de Estimação Generalizadas (GEE) é utilizado, a fim de verificar se houve alguma mudança na inadimplência das carteiras das cooperativas de crédito que pudesse ser atribuída à transformação das cooperativas de crédito para a modalidade de livre admissão, ao sistema cooperativista de crédito a que pertença ou ao seu tamanho.

\section{O SISTEMA FINANCEIRO}

Assim como ocorre com outros tipos de empresa, a organização e o funcionamento das instituições financeiras têm sido regulamentados por normativos específicos. Segundo Saunders (2000), a justificativa dos defensores dessa regulamentação apoia-se no fato de que as instituições financeiras exercem funções e prestam serviços que são muito importantes para a economia, influenciando o seu crescimento.

Para Cetorelli e Strahan (2006), a pesquisa econômica, em anos recentes, tem focado intensamente o papel desempenhado pelos mercados financeiros em relação à economia real. Segundo os autores, diversos estudos têm provido de evidência empírica que mercados financeiros amplos e bem desenvolvidos estão fortemente relacionados causalmente com o crescimento econômico futuro.

Diamonde Dybvig(2000)demonstraram três importantes pontos acerca do funcionamento do sistema financeiro:

a. Os depósitos das instituições financeiras promovem um mercado mais competitivo, mitigando o risco existente;

b. Por outro lado, esses mesmos depósitos também poderão levar à "corrida aos 
bancos", na qual todos os depositantes, incluindo os que prefeririam deixar seus depósitos se não estivessem preocupados com a quebra do banco, entram em pânico e tentam sacar seus depósitos imediatamente, levando o banco à falência;

c. Essa "corrida aos bancos" pode causar problemas econômicos sérios, porque a falência dos bancos causaria a quebra dos contratos dos empréstimos e o término dos investimentos produtivos.

Existem muitas situações nas quais os personagens que interagem com as instituições financeiras precisam de uma proteção maior. Um exemplo óbvio seria a falência de um banco que pode afetar todo o sistema financeiro. (MACEY E O'HARA, 2003).

É por isso que Diamond e Rajan (2000) observam que as instituições financeiras tem que lidar com a difícil escolha entre emprestar e criar liquidez e os custos de uma eventual corrida ao banco.

Também seria por isso que as autoridades monetárias estabelecem requerimentos de capital mínimo para as instituições financeiras, especificando a razão mínima entre o capital e o total de ativos necessários para que uma instituição financeira continue a operar, tornando-a mais segura. Por outro lado, um aumento dos requerimentos de capital causa restrições de crédito (DIAMOND e RAJAN, 2000).

Os autores também analisam os efeitos dos fundos garantidores de depósitos. Eles afirmam que, no caso de todos os depósitos serem segurados, não existirá, essencialmente, nenhuma diferença entre os depósitos e o capital. Dessa forma, os bancos estariam mais seguros, mas não criariam liquidez.

Para Boyd e De Nicoló (2005), os modelos modernos de risco bancário consideram o papel dos fundos garantidores de depósitos e outras intervenções governamentais que resultam em risco moral, distorcendo os incentivos aos riscos bancários, resultando em incentivo a uma intencional tomada de risco.

Ainda sobre o funcionamento do sistema financeiro, Diamond e Rajan (2001) observam que as instituições financeiras emprestam para tomadores ilíquidos e, ao mesmo tempo, provêm liquidez na demanda dos depositantes. Dessa forma, as instituições financeiras criam riscos de liquidez. É por isso que a instituição financeira não pode emprestar sem exigir um prêmio por essa falta de liquidez.

Gorton e Pennachi (1990) observam que as perdas associadas com a assimetria de informações podem ser mitigadas pelas garantias apresentadas, viabilizando a sua comercialização. Dessa forma, as instituições financeiras forneceriam um meio transacional menos arriscado.

Para Diamond (1984), as instituições financeiras possuem uma grande vantagem em monitorar os contratos dos empréstimos firmados com os tomadores de empréstimos, devido a diversificação de sua carteira.

Em uma economia em que todos são neutros ao risco a diversificação seria importante, porque aumentaria a probabilidade de a instituição financeira ter recursos suficientes para honrar seus compromissos com um depositante qualquer. No limite, a diversificação faria com que a 
probabilidade de insolvência da instituição financeira tenda a zero.

Já em uma economia em que todos são avessos ao risco, a instituição financeira teria menores custos de monitoração do que um investidor qualquer, o que aumentaria a sua tolerância. Dessa forma, uma instituição financeira que monitora muitos empreendimentos com projetos independentemente distribuídos cobraria um prêmio de risco menor do que um investidor qualquer (DIAMOND, 1984).

Diamond (1991) observa que a monitoração poderá falhar na provisão de incentivos para eliminar o risco moral, bem como poderá destruir o seu próprio valor, devido aos efeitos da reputação. Isso porque, em períodos de altas taxas de juros ou de baixas lucratividades, uma classificação de crédito mais alta é requerida para um empréstimo sem monitoração.

Dessa forma, a reputação é importante quando existe um conjunto de características exógenas relevantes em um grupo observável de agentes, porque isso implica que existe muito a se aprender sobre um agente (DIAMOND, 1989).

A seleção adversa torna-se menos severa quando são produzidos registros mais longos. Dessa forma, quando um tomador de empréstimo alcança uma boa reputação no longo prazo, a taxa de juros cai e o valor presente dos rendimentos projetados aumenta (DIAMOND, 1989).

\subsection{O cooperativismo de crédito}

Segundo Pinheiro (2007), a primeira cooperativa no mundo foi a Sociedade dos Probos Pioneiros, fundada em 21 de dezembro de 1844, na cidade de Rochdale, Inglaterra.

Já a primeira cooperativa de crédito foi fundada em 1856 em Delitzsch na Alemanha (PINHEIRO, 2007). As primeiras cooperativas de crédito rural também surgiram na Alemanha, por iniciativa de Friedrich Wilhelm Raiffeisen, que fundou as chamadas "Caixas de Crédito Raiffeisen”. Ainda hoje este tipo de cooperativa é bastante popular naquele país, tendo influenciado, sobremaneira, a constituição e o funcionamento das cooperativas de crédito rural brasileiras.

Na Europa, em especial na Alemanha, na França e na Holanda, o cooperativismo de crédito têm sido utilizado como instrumento impulsionador de setores econômicos estratégicos, em especial a agricultura (ALVES e SOARES, 2003). Além disso, destacam-se também as experiências americanas e canadenses no setor.

Na França, o Crédit Agrícole (CA), fundado em 1894 foi considerado a maior instituição financeira francesa, a $2^{\mathrm{a}}$ da Europa e $8^{\mathrm{a}}$ do mundo, englobando 39 bancos regionais e 2.533 cooperativas de crédito locais. No final de 2010, o grupo possuía cerca de 11.500 agências de atendimento, 54 milhões de clientes, mais de 160 mil empregados, 1,59 trilhões de euros em ativos e 500 bilhões de euros em empréstimos (CRÉDIT AGRÍCOLE, 2011).

Na Alemanha, berço do cooperativismo de crédito, o setor também tem se destacado, possuindo, em maio de 2010, cerca de 16,5 milhões de cooperados, 1,2 trilhões de euros em ativos, correspondendo por cerca $27,1 \%$ do total de empréstimos para pequenas e médias 
empresas e 28,9\% do total de depósitos (DGRV, 2010).

Na Holanda o cooperativismo de crédito é representado pelo Rabobank Nederland. Segundo seu Relatório Anual de 2008 (Rabobank Group Annual Sustainability Report 2008), essa instituição financeira é a maior provedora de serviços financeiros no mercado holandês, compreendendo 153 cooperativas de crédito locais, uma organização central e um grande número de subsidiárias especializadas. Atualmente, possui 9,5 milhões de clientes, 60.568 empregados, ativos de 612 bilhões de euros e lucros de 2,7 bilhões de euros, respondendo por cerca $39 \%$ do total de empréstimos para pequenas e médias empresas e $43 \%$ do total de depósitos (RABOBANK, 2009). No entanto, o cooperativismo de crédito também tem colhido resultados expressivos em diversos países em desenvolvimento.

No caso específico da América Latina, Westley e Branch (2000) observam que os empréstimos e os depósitos das cooperativas de crédito respondem por apenas de $1 \%$ a 3\% do total dos sistemas financeiros contra $10 \%$ a $20 \%$ em muitos países desenvolvidos.

Apesar disso, os autores defendem que as cooperativas de crédito são importantes na América Latina porque elas são a maior fonte de crédito para os microempreendedores da região. Além disso, possuem ainda um grande potencial de expansão e crescimento. Outro aspecto destacado pelos autores se baseia na capacidade das cooperativas de crédito de atender grandes populações menos favorecidas.

A história do cooperativismo de crédito no Brasil inicia-se em 28 de dezembro de 1902, com a constituição, em Nova Petrópolis/RS, da primeira cooperativa de crédito brasileira - a "Caixa de Economia e Empréstimos Amstad", que era do "do tipo Raiffensen" (PINHEIRO, 2007), que continua em atividade até os dias de hoje, sob a denominação de "Cooperativa de Crédito de Livre Admissão de Associados Pioneira da Serra Gaúcha - SICREDI Pioneira RS”. Ressalta-se que esta cooperativa passou a ser da modalidade de livre admissão por decisão da Assembléia Geral Extraordinária realizada em 17 de abril de 2007.

Segundo Bittencourt (2001), as cooperativas de crédito, até a década de 1960, exerceram um papel financeiro importante em muitos municípios brasileiros. Apesar disso, muitas delas começaram a enfrentar sérios problemas administrativos, o que levou a uma situação de desconfiança e de desvirtuamento de seus propósitos iniciais.

A Lei da Reforma Bancária (Lei 4.595/64) marcou o início de um novo arcabouço legal para o sistema cooperativista de crédito no Brasil, traduzindo-se, especialmente, pela criação do Banco Central do Brasil (BACEN), pela equiparação das cooperativas de crédito às demais instituições financeiras e pela atribuição ao BACEN de autorizar e fiscalizar estas cooperativas (PINHEIRO, 2007).

Por meio de acesso ao sistema Unicad - Informações sobre Entidades de Interesse do Banco Central, constatou-se que o sistema cooperativista de crédito no Brasil era composto, em 31 de dezembro de 2010, de 2 bancos cooperativos, 4 confederações, 1 federação, 38 cooperativas centrais em funcionamento e 1.317 cooperativas de crédito singulares em funcionamento, somando cerca de 5 milhões de associados. As quatro confederações de crédito correspondem ao Sistema de Crédito Cooperativo (Sicredi), ao Sistema de Cooperativas de Crédito do Brasil (Sicoob Brasil), a Unicred do Brasil e a Confebrás. 
Em 31 de dezembro de 2010, a participação das cooperativas de crédito representava 2,0\% dos depósitos e 2,3\% das operações de crédito, tomando-se como base o total do Sistema Financeiro Nacional (SFN). Segundo Soares e Melo Sobrinho (2007), apesar dos números ainda modestos, os autores destacam que o cooperativismo de crédito, em especial no sul do Brasil, tem contribuído para o fortalecimento da economia local em perfeita harmonia com seus objetivos.

\subsection{Desempenho de cooperativas de crédito}

Apesar de Cuevas e Fischer (2006) afirmarem que as cooperativas de crédito estão entre as instituições financeiras menos estudadas, já existem trabalhos que tiveram como foco principal o desenvolvimento do cooperativismo de crédito, bem como o desempenho destas instituições financeiras.

Ferguson e McKillop (1997), por exemplo, criaram uma tipologia com o objetivo de classificar os diferentes estágios de desenvolvimento do cooperativismo de crédito nos diversos países. Segundo este critério, os estágios de desenvolvimento podem ser classificados como: indústria nascente; indústria em transição; e indústria madura.

No entanto, Sibbald, Ferguson e McKillop (2002) concluem que não há um caminho único a seguir para que o cooperativismo de crédito se desenvolva, o que demonstra a complexidade de se propor uma tipologia para classificar o desenvolvimento desta indústria.

Ward e McKillop (2005) investigaram a ligação entre as características e localização de cooperativas de crédito com seu sucesso. Para isso, tamanho, idade, filiação a um sistema, existência de vínculo de associação e localização foram consideradas variáveis importantes para o sucesso de uma cooperativa de crédito. Os autores identificaram uma relação positiva estatisticamente significante entre a variável tamanho da cooperativa e seu sucesso, indicando a existência de economias de escala na amostra estudada. Também foi encontrada uma relação positiva estatisticamente significante entre a variável idade e o seu sucesso. Já a variável filiação a um sistema teve resultados contraditórios, visto que foi obtida uma relação positiva estatisticamente significante para um sistema e uma relação negativa para outro sistema. Finalmente, há uma relação positiva estatisticamente significante entre a variável existência de vínculo de associação e o sucesso da cooperativa, o que foi atribuído a menores custos operacionais, devido ao melhor conhecimento de seus associados.

Ao estudar o crescimento das cooperativas de crédito norte americanas na década de 1990, Goddard, McKillop e Wilson (2002) também encontraram uma relação positiva estatisticamente significante entre a variável tamanho e o crescimento da cooperativa, sugerindo que as cooperativas de crédito maiores crescem, na média, mais rápido do que as menores. Por outro lado, em relação à variável idade, apurou-se que as cooperativas de crédito mais jovens tendem a crescer mais rápido do que as mais velhas.

Para mensurar o desempenho de cooperativas de crédito, Fried, Lovell e Eeckaut (1993) fizeram uso de um modelo comportamental por considerarem essas instituições financeiras 
diferentes dos bancos comerciais, visto que não possuem fins lucrativos. Por isso, consideraram que o objetivo dessas instituições seria maximizar os benefícios a seus associados, o que foi expresso pela maximização dos serviços disponibilizados em função dos recursos disponíveis e do ambiente operacional em que atuam.

Westley e Shaffer (1999) procuraram mensurar o desempenho de cooperativas de crédito na América Latina. Segundo os autores, os lucros (sobras, no caso de todos os tipos de cooperativas) são importantes para as cooperativas de crédito, assim como o são para as demais instituições financeiras, porque auxiliam na construção de seu capital social, o que poderá ser utilizado em futuras expansões ou servirá como um colchão de liquidez em eventuais crises, promovendo, portanto, a sua sobrevivência no longo prazo.

Além disso, os autores ressaltam que a inadimplência tem sido a causa mais importante das perdas e da insolvência das cooperativas de crédito. Alertam que uma inadimplência acima de 5 a $10 \%$ já causaria um impacto significativo nas receitas e aumentaria os custos administrativos.

Como resultado de um trabalho patrocinado pela United States Agency for International Development (USAID), entre 1987 e 1994, na Guatemala, foram propostos diversos indicadores de desempenho específicos para o cooperativismo de crédito (Richardson IN Westley e Branch, 2000) entre os quais destaca-se a inadimplência, medida com base na relação entre o total das operações inadimplentes há mais de 30 dias (Inadimplência $>30$ dias) e o total de operações de crédito (Empréstimos), cujo valor aceitável é menor do que 10\%.

Neste artigo, eventuais mudanças do desempenho das cooperativas de crédito serão analisadas a partir da relação entre a inadimplência e as seguintes variáveis preditoras: livre admissão, variável dummy utilizada para determinar quando a cooperativa de crédito se transformou para a modalidade de livre admissão; sistema cooperativista de crédito; tamanho da cooperativa; e tempo.

\section{METODOLOGIA}

A pesquisa tem como unidades de análise o universo de todas as 56 cooperativas de crédito que se transformaram para a modalidade de livre admissão antes de 31/12/2005 e que não incorporaram outra cooperativa de crédito. Essa data de corte se justifica para que se tenha pelo menos 18 meses de análise após a transformação de cada uma dessas cooperativas de crédito. Cabe ressaltar que as cooperativas de crédito estudadas pertencem a dois sistemas cooperativistas de crédito distintos, a saber: SICOOB e SICREDI.

Optou-se por aplicar o Modelo de Equações de Estimação Generalizadas (GEE) devido a necessidade de se analisar as relações entre uma variável independente e muitas variáveis preditoras. Além disso, o GEE é o modelo adequado para a análise de dados longitudinais em populações (FITZMAURICE, LAIRD e WARE, 2004). Isso significa que por meio dele é possível avaliar os impactos das variáveis considerando o conjunto das cooperativas de crédito estudadas, e não apenas as cooperativas individualmente. Essa propriedade é importante, pois 
as conclusões obtidas nesse trabalho poderão ser generalizadas para as cooperativas de crédito que se transformaram para a modalidade de livre admissão. Logo, o GEE não seria adequado se o interesse fosse avaliar o efeito das variáveis respostas em cada empresa, individualmente.

O GEE não requer nenhuma premissa sobre a distribuição para as observações. Dessa forma, o GEE provê um método unificado para analisar diferentes tipos de dados longitudinais sem fazer suposições sobre a distribuição do vetor de respostas, sendo necessárias apenas suposições acerca da modelagem de suas médias (FITZMAURICE, LAIRD e WARE, 2004).

O GEE é um método iterativo que utiliza quase-verosimilhança (WEDDERBURN, 1974) para estimar os coeficientes da regressão. Detalhes sobre quase-verosimilhança podem ser encontrados em extensa literatura (McCULLAGH, 1983; NELDER e PREGIBON, 1987; ZEGER e QAQISH, 1988; NELDER e LEE, 1992; DIGGLE, LIANG e ZEGER, 1994).

O ajuste de Modelo do tipo GEE envolve a escolha de uma matriz de correlação que represente a associação entre as empresas e entre as observações de cada empresa. Segundo Twisk (2003), a melhor escolha para esta matriz deve levar em conta sua qualidade para representar os dados e sua parcimônia. Como o banco de dados disponível possui um grande número de observações para cada cooperativa de crédito, para a utilização de uma matriz nãoestruturada seria necessário estimar um número muito grande de parâmetros. Assim, com o objetivo de aumentar o poder do teste e, ao mesmo tempo, garantir que os dados sejam bem representados, optou-se pela utilização de uma matriz de correlação uniforme.

Desenvolvido por Liang e Zeger (1986), o Modelo de Equações de Estimação Generalizadas (GEE) é uma alternativa para o ajuste de modelos de regressão quando os dados disponíveis são longitudinais. A partir desse método, é possível encontrar parâmetros consistentes e assintoticamente normais mesmo que a matriz de correlação dos dados seja incorretamente especificada.

Assim sendo, deve-se supor que uma cooperativa de crédito $i, i=1, \ldots, n$, será observada $t_{i}$ vezes ao longo do tempo. Nesse caso, $y_{i j}$ e $x_{i j}$ serão, respectivamente, o valor observado da variável resposta e da variável explicativa para a cooperativa de crédito $i$, observação $j$ com $j$ $=1, \ldots, t_{i}$. Pode-se observar que, $x_{i j}=\left(x_{i j 1}, x_{i j 2}, \ldots, x_{i j p}\right)^{T}$ será um vetor quando o modelo de interesse apresentar mais de uma variável explicativa.

Além disso, considera-se $E\left(y_{i j}\right)=\mu_{\mathrm{ij}}$ e $\operatorname{Var}\left(y_{i j}\right)=\phi^{-1} v\left(\mu_{\mathrm{ij}}\right) \phi^{-1} v_{i j} i=1,2, \ldots, n$. A modelagem da média leva em conta a função de ligação $g\left(\mu_{\mathrm{tj}}\right)$ na seguinte forma:

$$
g\left(\mu_{i j}\right)=\sum_{k=1}^{p} x_{i j k} \beta_{k}
$$

Em que $g: R->R$ é uma função inversível e duplamente diferenciável e $\beta=\left(\beta_{1}, \ldots, \beta_{\mathrm{p}}\right)$ Té um vetor paramétrico real.

Admita-se que a matriz de correlação de trabalho $\Gamma\left(u_{\mathrm{i}}\right), \operatorname{com} u_{\mathrm{i}}=\mathrm{y}_{\mathrm{i}}-\mu_{\mathrm{i}}$ seja desconhecida. Nesse caso será necessário utilizar uma função de estimação que considere um estimador de $\Gamma\left(u_{\mathrm{i}}\right)$, definido pelo vetor paramétrico $\alpha$. Essa função é obtida por meio da introdução de $\alpha$ 
em uma adaptação daquela utilizada para estimar os parâmetros dos chamados Modelos Lineares Generalizados (GLM). O GLM, assim como o GEE, considera uma função de ligação na modelagem da média, embora seja específico para dados independentes (NELDER e WEDDERBURN, 1972). Assim, os parâmetros serão obtidos a partir de:

$$
\Psi_{n}(\beta)=\sum_{i=1}^{n} D_{i}^{T} W_{i}^{-1}\left(\hat{\alpha}_{n}\right) u_{i}
$$

Em que $D_{i}^{T}=X_{i}^{T} H_{i}, \mathrm{H}_{\mathrm{i}}=\vartheta \mu_{\mathrm{i}} / \vartheta \eta_{i}, W=\operatorname{Cov}\left(u_{i}\right)=\phi^{-1} \mathrm{~A}_{\mathrm{i}}^{1 / 2} \Gamma\left(u_{\mathrm{i}}\right) \mathrm{A}_{\mathrm{i}}^{1 / 2}$ e $\mathrm{A}_{\mathrm{i}}=\operatorname{diag}$ $\left\{v_{i}, \ldots, v_{n}\right\}$.

O parâmetro de dispersão $\phi^{-1}$ apresentado no modelo corresponde a um parâmetro de perturbação. Assumindo, sem perda de generalidade, $t_{i}=t$, se o quarto momento de $y_{i j}$ é finito, demonstra-se que:

$$
\hat{\phi}^{-1}=\left\{\sum_{i=1}^{n} \sum_{j=1}^{t} \hat{r}_{i j}^{2} /(n t-p)\right\}^{-1}
$$

é um estimador $\sqrt{n}$ - consistente de $\phi^{-1}$ quando $\hat{r}_{i j}$ é um estimador do resíduo de Pearson para a observação $\mathrm{y}_{\mathrm{ij}}$ definido como:

$$
\hat{r}_{i j}=\frac{y_{i j}-\hat{\mu}_{i j}}{\left\{v_{i j}\right\}^{1 / 2}}
$$

Como neste trabalho a matriz de correlação de trabalho utilizada foi a matriz uniforme, assumiu-se que a correlação entre quaisquer duas observações de um mesmo indivíduo é a mesma. Assim, a matriz de correlação de trabalho será:

$$
\Gamma\left(\alpha_{\mathrm{ij}}\right)=\left[\alpha_{\mathrm{ij}}\right] i, j=1, \ldots \mathrm{t}
$$

com $\alpha_{\mathrm{ii}}=1$ e $\alpha_{\mathrm{ij}}=\alpha$. Um estimador consistente para esse parâmetro, dado $\phi$ será $\quad$ (LAIRD e WARE, 1982):

$$
\hat{\alpha}=\phi \frac{\sum_{i=1}^{n} \sum_{j>k} \hat{r}_{i j} \hat{r}_{i k}}{\sum_{i=1}^{n} \frac{1}{2} t(t-1)-p}
$$

Finalmente, para estimação dos parâmetros do modelo (1) deve-se utilizar um método iterativo, mais explicitamente, o método modificado de Newton (JORGENSEN et al., 1996). Seja $(l+1)$ o passo em que se encontra o processo, o valor do parâmetro nesse ponto será:

$$
\beta^{(l+1)}=\beta^{(l)}-\mathrm{S}^{-1}\left(\beta^{(l)}\right) \psi_{n}\left(\beta^{(l)}\right)
$$


Em que $S=\sum_{i=1}^{\prime \prime} S_{i}\left(\beta^{(l)}\right) \quad \operatorname{com} S_{i}=S_{i}^{T} W_{i}^{-1} D_{i}$.

Para cada modelo ajustado, foi necessário excluir as informações das cooperativas de crédito que possuíam dados inexistentes para pelo menos uma das variáveis envolvidas no modelo. Nesse caso, optou-se por não utilizar um método de estimação desses dados inexistentes, uma vez que tal procedimento poderia resultar em uma perda de qualidade do modelo.

Dessa forma, foi ajustado um modelo em que a variável resposta é a inadimplência e as variáveis preditoras serão livre admissão, sistema, tamanho e tempo.

Ressalta-se que o objetivo principal é avaliar se houve uma mudança de desempenho de cada indicador ao longo do tempo e depois de a cooperativa de crédito se tornar de livre admissão, levando-se em consideração os sistemas cooperativistas de crédito. Dessa forma, as variáveis livre admissão, sistema e tempo serão sempre forçadas a entrar no modelo.

Logo, será necessário apenas ponderar sobre a qualidade de dois modelos distintos: com a variável tamanho ou sem ela. Para isso, utilizou-se um nível de significância de 5\% para o Teste de Wald. O teste de Wald possui como hipóteses:

$$
\left\{\begin{array}{l}
H_{o}: \beta_{\text {tamanho }}=0 \\
H_{a}: \beta_{\text {tamanho }} \neq 0
\end{array}\right.
$$

A definição do tamanho da cooperativa foi feita levando-se em conta o patrimônio líquido (PL) de cada cooperativa na data base de 12/2005, com base nos seguintes pontos de corte:

- pequena cooperativa de crédito de livre admissão: $\mathrm{PL}<\mathrm{R} \$ 5$ milhões

- média cooperativa de crédito de livre admissão: $\mathrm{R} \$ 5$ milhões $\leq \mathrm{PL}<\mathrm{R} \$ 10$ milhões

- grande cooperativa de crédito de livre admissão: PL $\geq R \$ 10$ milhões

Por essa classificação, foi obtida a seguinte distribuição para as 56 cooperativas de crédito estudadas:

- 27 pequenas cooperativas de crédito de livre admissão;

- 15 médias cooperativas de crédito de livre admissão; e

- 14 grandes cooperativas de crédito de livre admissão.

A equação seguinte representa, de maneira genérica, os modelos que serão ajustados:

Inadimplência $=\beta_{0}+\beta_{1}$ liv.adm $+\beta_{2}$ sistema $+\beta_{3}$ coop.média $+\beta_{4}$ coop.grande $+\beta_{5}+\varepsilon_{\mathrm{t}}$

em que:

Inadimplência é o indicador a ser avaliado

$\beta_{0}$ é o intercepto 
$\beta_{1}$ : são os coeficientes das variáveis utilizadas

liv.adm: é uma variável dummy, tal que:

- liv.adm. = 1,após a cooperativa de crédito se transformar para a modalidade de livre admissão;

- liv.adm. $=0$, antes da cooperativa de crédito se transformar.

Sistema: é uma variável dummy, tal que:

- $\quad$ sistema $=1$, caso a cooperativa de crédito seja filiada ao Sistema SICOOB

- $\quad$ sistema $=0$, caso a cooperativa de crédito seja filiada ao Sistema SICREDI

coop.média : é uma variável dummy, tal que:

- coop.média $=1$, se R $\$ 5$ milhões $\leq \mathrm{PL}<\mathrm{R} \$ 10$ milhões em 12/2005

- coop.média $=0$, caso contrário

coop.grande : é uma variável dummy, tal que:

- coop.grande $=1$, se PL $\geq \mathrm{R} \$ 10$ milhões em 12/2005

- coop.grande $=0$, caso contrário

$t$ :é o tempo (em meses) e

$\varepsilon_{i}$ : é o erro da regressão

Para representar as três categorias de tamanhos de cooperativas de crédito foram utilizadas duas variáveis dummy, coop. média e coop. grande. A categoria pequena cooperativa de crédito é considerada como de referência. Dessa forma, os tamanhos de uma cooperativa de crédito são representados como:

\begin{tabular}{|l|c|c|}
\hline & coop. média & coop. grande \\
\hline Pequena cooperativa de crédito de livre admissão & 0 & 0 \\
\hline Média cooperativa de crédito de livre admissão & 1 & 0 \\
\hline Grande cooperativa de crédito de livre admissão & 0 & 1 \\
\hline
\end{tabular}

Quadro 1: Representação do tamanho das cooperativas de crédito no modelo

Fonte: elaborado pelos autores

Assim, o coeficiente encontrado para a variável coop. média refere-se à variação encontrada na variável resposta quando a cooperativa de crédito de livre admissão é classificada como de médio porte em relação às de pequeno porte (categoria de referência). Já o coeficiente para o coop. grande é referente à variação observada na variável resposta quando a cooperativa de crédito de livre admissão é classificada como de grande porte em relação às de pequeno porte.

As fontes de dados utilizadas são os balancetes enviados mensalmente pelas cooperativas de crédito ao Banco Central do Brasil. A pesquisa é feita, portanto, a partir de dados secundários.

Cabe ressaltar que os registros contábeis das instituições financeiras brasileiras são 
feitos de acordo com o Plano Contábil das Instituições do Sistema Financeiro Nacional (Cosif), o que garante a existência de um padrão único e conhecido (BACEN: 1987). Além disso, todas as análises foram feitas utilizando-se o pacote estatístico R, versão 2.5.1.

\section{RESULTADOS}

Inicialmente, foram calculadas as medidas de tendência central e de dispersão das taxas de inadimplência, tais como a média, a mediana, o desvio-padrão e a variância, o que viabilizou a comparação dos resultados encontrados com os valores considerados ideais. Com isso, é possível traçar um perfil global das cooperativas de crédito de livre admissão, levando-se em consideração os seus respectivos sistemas cooperativas de crédito.

Tabela 1: Medidas de tendência central

\begin{tabular}{c|c|c|c|c}
\hline Indicador & Sistema & \multicolumn{1}{c|}{ Média } & \multicolumn{1}{c}{ Mediana } & Desvio-Padrão \\
\hline \multirow{2}{*}{ Inadimplência (\%) } & SICREDI & 2,42 & 1,71 & 2,41 \\
& SICOOB & 3,56 & 2,8 & 3,85 \\
\hline
\end{tabular}

Fonte: elaborado pelos autores

Cabe ressaltar que para o cálculo deste indicador foram consideradas inadimplentes todas as operações de crédito em atraso há mais de 30 dias. O valor aceitável é menor do que $10 \%$, o que foi alcançado em média pelas cooperativas de crédito de ambos os sistemas.

Uma metodologia diferente para a inadimplência foi utilizada pelo Banco Central no Relatório de Estabilidade Financeira de novembro de 2007 que considerou inadimplente uma operação de crédito que tenha parcelas em atraso há mais de 90 dias. Por esta metodologia, a inadimplência no sistema financeiro nacional era de 3,8\% em junho de 2007 (BACEN, 2007).

A seguir, é avaliado o ajuste do modelo de Equações de Estimação Generalizadas (GEE) para a inadimplência.

Tabela 2: Modelo GEE para a inadimplência

\begin{tabular}{ccccc}
\hline \hline Variável & $\beta_{\mathrm{i}}$ & Desvio Padrão $\left(\beta_{\mathrm{i}}\right)$ & Wald & P-valor \\
\hline intercepto $\left(\beta_{0}\right)$ & 0,025 & 0,005 & 29,558 & 0,000 \\
sistema & 0,012 & 0,006 & 4,495 & 0,034 \\
livre admissão & 0,007 & 0,003 & 5,911 & 0,015 \\
tempo & 0,000 & 0,000 & 0,827 & 0,363 \\
\hline \hline
\end{tabular}

Fonte: elaborado pelos autores

De acordo com o Teste de Wald realizado (p-valor igual a 0,40092), as variáveis referentes ao tamanho da cooperativa de crédito não foram consideradas significativas, e por isso não foram incluídas no modelo acima.

Dessa forma, a melhor regressão obtida para a inadimplência foi:

Taxa de Inadimplência $=\beta_{0}+\beta_{1}$ liv.adm. $+\beta_{2} \mathrm{t}+\varepsilon_{\mathrm{t}}$ 
O modelo ajustado indica que o indicador $\mathrm{i}_{6}$ não recebe influência da variável tempo a $5 \%$ de significância. Por outro lado, este indicador é significantemente afetado pelas variáveis livre admissão (p-valor igual a 0,034) e sistema (p-valor igual a 0,015$)$.

A partir do coeficiente da variável livre admissão, é possível concluir que a transformação das cooperativas de crédito para a modalidade de livre admissão ocasiona um aumento esperado de 0,007 unidade (ou seja, $0,7 \%$ ) no indicador $\mathrm{i}_{6}$. Pode-se concluir que foi observado um aumento da inadimplência, medido pela relação entre o total das operações inadimplentes há mais de 30 dias e o total de operações de crédito para estas cooperativas de crédito. Tal constatação pode ser considerada uma piora no desempenho neste indicador.

Além disso, adotar o fato de uma cooperativa de crédito de livre admissão pertencer ao SICOOB aumenta o valor esperado para a sua inadimplência $\left(i_{6}\right)$ em 0,012 unidades, ou seja, $1,2 \%$.

\section{CONCLUSÕES}

O objetivo principal deste artigo foi avaliar possíveis mudanças na inadimplência das carteiras de crédito das cooperativas de crédito que se transformaram para a modalidade de livre admissão, criada pela Resolução CMN 3.106/03, levando-se em consideração os sistemas cooperativistas de crédito e o tamanho das cooperativas de crédito. Para isso, foi ajustado um Modelo de Equações de Estimação Generalizadas (GEE) que considerassem a estrutura longitudinal dos dados e utilizassem como variáveis explicativas: a transformação para livre admissão (livre admissão), o sistema cooperativista de crédito ao qual cada cooperativa é filiada (sistema), o seu respectivo tamanho (coop. média e coop. grande) e o tempo (tempo).

Os modelos GEE ajustados identificaram mudança de desempenho depois da transformação para a modalidade de livre admissão para a inadimplência. Com relação a este indicador, observou-se um desempenho pior depois da transformação das cooperativas de crédito para aquela modalidade.

Esta constatação vai ao encontro dos resultados obtidos por Ward e McKillop (2005) que identificaram uma relação positiva estatisticamente significante entre a existência do vínculo de associação e o sucesso da cooperativa, o que foi atribuído pelos autores a menores custos operacionais, devido ao melhor conhecimento de seus associados.

Particularmente, a constatação de que há uma relação negativa estatisticamente significante entre a inadimplência e a transformação das cooperativas de crédito para a modalidade de livre admissão deveria ser objeto de preocupação por parte daquelas instituições financeiras, visto que Westley e Shaffer (1999) afirmam que a inadimplência tem sido a causa mais importante das perdas e da insolvência das cooperativas de crédito.

Por outro lado, deve ser ressaltado que as taxas de inadimplência observadas nas cooperativas de crédito estudadas se encontram em patamares bem inferiores a 5\%. Este é o limite a partir do qual, segundo os autores, já seria observado um impacto significativo nas 
receitas e um aumento de seus custos administrativos.

Levando-se em consideração a importância do tema e tendo em vista os resultados obtidos neste trabalho, sugere-se que essas cooperativas sejam estudadas aplicando-se outros modelos, por exemplo: o modelo comportamental utilizado por Fried, Lovell e Eeckaut (1993), bem como outros indicadores financeiros.

\section{REFERÊNCIAS}

ALVES, Sérgio Darcy da Silva, SOARES, Marden Marques. Microfinanças: Democratização do crédito no Brasil - Atuação do Banco Central. Brasília: Banco Central do Brasil, 2006.

ALVES, Sérgio Darcy da Silva, SOARES, Marden Marques. Nota Técnica: As cooperativas de crédito e o Banco Central do Brasil. Brasília: Banco Central do Brasil, 2003.

BANCO CENTRAL DO BRASIL (BACEN). Plano contábil das instituições do sistema financeira nacional - COSIF. Instituído pela Circular 1.273, de 29 de dezembro de 1987, para adoção obrigatória pelas instituições financeiras brasileiras a partir do Balanço de 30 de junho de 1988. Disponível em http://www.bcb.gov.br/mPag.asp?perfil=1\&cod=754\&codP=106\&idi oma $=$ P.

BANCO CENTRAL DO BRASIL (BACEN). Relatório de estabilidade financeira. Brasília: Banco Central do Brasil, Novembro 2007.

BANCO CENTRAL DO BRASIL. Resolução 2.682, de 31 de dezembro de 1999. Dispõe sobre critérios de classificação das operações de crédito e regras para constituição de provisão para créditos de liquidação duvidosa. Diário Oficial da União, Brasília: 31.12.1999.

BITTENCOURT, Gilson Alceu. Cooperativas de crédito solidário: constituição e funcionamento. 2.ed. Brasília: Estudos NEAD 4 (Núcleo de Estudos Agrários e Desenvolvimento Rural do Ministério do Desenvolvimento Agrário), 2001.

BOYD, J. H., DE NICOLÓ, G. The theory of bank risk taking and competition revisited. The Journal of Finance, June 2005, vol. LX, n. 3, 1329-1343, 15p.

BRASIL. Lei ${ }^{\circ}$ 4.595, de 31 de dezembro de 1964. Dispõe sobre a Política e as Instituições Monetárias, Bancárias e Creditícias, Cria o Conselho Monetário Nacional e dá outras providências. Brasília: Diário Oficial da União, 31.12.1964.

BRASIL. Lei $n^{\circ}$ 5.764, de 16 de dezembro de 1971. Define a Política Nacional de Cooperativismo, institui o regime jurídico das sociedades cooperativas, e dá outras providências. Brasília: Diário Oficial da União, 16.12.1971.

CETORELLI, N., STRAHAN, P. E. Finance as a barrier to entry: bank competiotion and industry structure in local U.S. markets. The Journal of Finance, February 2006, vol. LXI, 
n. $1,437-461$.

CONSELHO MONETÁRIO NACIONAL (CMN). Resolução 2.682, de 31 de dezembro de 1999. Dispõe sobre critérios de classificação das operações de crédito e regras para constituição de provisão para créditos de liquidação duvidosa. Brasília: Diário Oficial da União, 31.12.1999b.

CONSELHO MONETÁRIO NACIONAL (CMN). Resolução 3.106, de 25 de junho de 2003. Aprova Regulamento que disciplina a constituição e o funcionamento de cooperativas de crédito. Brasília: Diário Oficial da União, 25.06.2003.

CONSELHO MONETÁRIO NACIONAL (CMN). Resolução 3.442, de 28 de fevereiro de 2007. Aprova Regulamento que disciplina a constituição e o funcionamento de cooperativas de crédito. Brasília: Diário Oficial da União, 1.3.2007.

CRÉDIT AGRÍCOLE. Disponível em http://www.credit-agricole.fr. Acesso em 3 set. 2007.

CUEVAS, Carlos E., FISCHER, Klaus P. Cooperative financial institutions: issues in governance, regulation, and supervision. Washington: The World Bank, 2006.

DGRV. Disponível em http://www.dgrv.org. Acesso em 11 set.2007.

DIAMOND, Douglas W. Financial intermediation and delegated monitoring. Review of Economic Studies, 1984, 393-414, 22p.

DIAMOND, Douglas W. Monitoring and reputation: The choice between bank loans and directly placed debt. Journal of Political Economy, 1991, vol, 99, n. 41, 689-721, 33 p.

DIAMOND, Douglas W. Reputation acquisition in debt markets. Journal of Political Economy, 1989, vol, 97, n. 4, 828-862, 35p.

DIAMOND, Douglas W., DYBVIG, Philip H. Bank runs, deposit insurance, and liquidity. Federal Reserve Bank of Minneapolis, Quarterly Review, Winter 2000, 14-23, 10p.

DIAMOND, Douglas W., RAJAN, Raghuram G. A theory of bank capital. The Journal of Finance, December 2000, vol. LX, n. 6, 2431-2465, 35p.

DIAMOND, Douglas W., RAJAN, Raghuram G. Liquidity risk, liquidity creation, and financial fragility: A theory of banking. Journal of Political Economy, 2001, v. 109, n. 29, 287-327, 41p.

DIGGLE, P.J., LIANG, K.Y., ZEGER, S.L. Analysis of Longitudinal Data. Repr. 1994, 1995 (with corrections). Oxford: Clarendon Press; New York: Oxford University Press.

FERGUSON. Charles, McKILLOP, Donal G. An industry approach to classifying credit union development. Working Paper. Coleraine: University of Ulster, 1997.

FISCHER, Klaus P. Financial Cooperatives: a "market solution” to SME and rural financing. 
Working Paper. Quebec: Centre de Recherche en Économic et Finance Apliquées (CRÉFA), 1998.

FITZMAURICE, Garrett M., LAIRD, Nan M., WARE, James H. Applied Longitudinal Analysis. Hoboken: John Wiley and Sons, 2004.

FRIED, Harold O., LOVELL, C. A. K., EECKAUT, Philippe V. Evaluating the performance of US credit unions. Journal of Banking and Finance 17 (1993) 251-265.

GODDARD, John A., McKILLOP Donal G., WILSON John O. S. Scale and life cycle effects on credit union growth: an empirical investigation. Working Paper. Swansea: University of WDiggales Swansea, 2002.

GORTON, G., PENNACCHI, G. Financial intermediaries and liquidity creation. The Journal of Finance, March 1990, vol. XLV, n. 1, 49-71, 23p.

JORGENSEN, B., LUNDBYE-CRISTENSEN, S., SONG, X. K., SUN, L. State space models for multivariate longitudinal data of mixed types. Canadian Journal of Statistics, 1996, 24, 385-402.

LAIRD, N. M.; WARE, J. H. Random effects models for longitudinal data. Biometrics, 1982, v.38, n. 4, 963-974.

LIANG, K. Y., ZEGER, L. S. Longitudinal data analysis using generalized linear models. Biometrika, 1986, v. 73, 13-22.

MACEY, J. R., O'HARA, M. The Corporate Governance of Banks. Economic Policy Review - Federal Reserve Bank of New York; Apr 2003; 9, 1; ABI/INFORM Global, p. 91-107.

McCULLAGH, P. Quasi-likelihood functions. Annals of Statistcs, 1983, v. 11, 59-67.

McKILLOP, Donal G. Financial cooperatives: structure, conduct and performance. Annals of Public \& Cooperative Economics, Sep. 2005, v. 76 Issue 3, 301-305.

MEINEN, Ênio, DOMINGUES, Jefferson N., DOMINGUES, Jane A. S. Cooperativas de crédito no direito brasileiro. Porto Alegre: Editora Sagra Luzzatto, 2002.

NELDER, J. A., LEE, Y. An extended quasi-likelihood and pseudo-likelihood: some comparasions. Journal of the Royal Statistical Society Series B, 1992, v. 54, 273-284.

NELDER, J. A., PREGIBON, D. An extended quasi-likelihood function. Biometrika, 1987, v. 74, 221-232.

NELDER, J. A., WEDDERBURN, R. W. M. Generalized Linear Models. Journal of the Royal Statistical Society Series A, 1972, v. 135, 370-384.

PINHEIRO, Marcos Antonio Henriques. Cooperativas de crédito: História da evolução 
normativa no Brasil. 5. ed. Brasília: Banco Central do Brasil, 2007.

RABOBANK, Annual responsability and sustainability report 2008. Utrecht: Rabobank Group, 2009.

SAUNDERS, Anthony M. Administração de instituições financeiras. São Paulo: Atlas, 2000.

SIBBALD, Alexander; FERGUSON, Charles; McKILLOP, Donal G. An examination of key factors of influence in the development process of credit union industries. Annals of Public \& Cooperative Economics, Sep. 2002, v. 73 Issue 3, 399-428.

SOARES, Marden Marques, MELO SOBRINHO, Abelardo Duarte. Microfinanças: O papel do Banco Central do Brasil e a importância do cooperativismo de crédito. Brasília: Banco Central do Brasil, 2007.

TWISK, J. W. R. Applied longitudinal data analysis for epidemiology: a practical guide. Cambridge, UK; New York: Cambridge University Press, 2003.

WARD, Ann-Marie, McKILLOP, Donal G. An investigation into the link between UK credit union characteristics, location and their success. Annals of Public \& Cooperative Economics, Sep. 2005, v. 76 Issue 3, 461-489.

WEDDERBURN, R. W. M. Quasi-likehood function, generalized linear models, and GaussNewton method. Biometrika, 1974, v. 61, 439-447.

WESTLEY, Glenn D., BRANCH, Brian (org.). Safe money: building effective credit unions in Latin America. Washington: Inter-American Development Bank and World Council of Credit Unions, 2000.

WESTLEY, Glenn D., SHAFFER, Sherrill. Credit union policies and performance in Latin America. Journal of Banking \& Finance, 1999, 23, 1303-1329.

ZEGER, S. L., QAQISH, B. Markov regression models for time series: a quasi-likelihood approach. Biometrics, 1988, v. 44, 1019-1031. 


\section{ENDEREÇO DOS AUTORES:}

\section{Romeu Eugênio Lima}

Banco Central do Brasil.

Av. Álvares Cabral, 1605

Santo Agostinho

Belo Horizonte, MG - Brasil

30170-001

\section{Hudson Fernandes Amaral}

Universidade Federal de Minas Gerais, Faculdade de Ciências Econômicas, Departamento de Ciências Administrativas.

Av. Antônio Carlos, 6627 - Prédio FACE - Bloco 01 - 4 andar - Sala 4030, Pampulha

Belo Horizonte, $\mathrm{MG}$ - Brasil

$31270-901$ 